\title{
QUADRATIC ALGEBRAS WITH FEW RELATIONS
}

\author{
by JAMES J. ZHANG
}

(Received 3 May, 1996)

0. Introduction Throughout $V$ will be a finite dimensional vector space over a field $k$ and $T(V)$ will denote the tensor algebra over $V$. For simplicity the symbol $\otimes$ will be omitted in the writing of the elements of $T(V)$. Let $\left\{x_{i}\right\}_{1}^{n}$ be a basis of $V$ ordered by $x_{i}<x_{i+1}$ for all $i$. Then we order the non-commutative monomials $\left\{x_{i_{1}} \ldots x_{i_{1}} \mid l \geq 0\right.$ and $1 \leq i_{s} \leq n$ for $\left.s=1, \ldots, l\right\}$ lexicographically from the left. D. Anick [1, p. 652] defines the high term of an element $b$ in $T(V)$ to be the highest monomial appearing in $b$. As a consequence of $[1,3.2]$, if the set of the high terms of homogeneous relations is combinatorically free in the sense of no overlap ambiguities, then the connected algebra has global dimension 2. The purpose of this note is to prove this result and more for quadratic algebras under other hypotheses on the relations.

Define the rank of $r \in V \otimes V$ to be the minimal $d$ such that $r=\sum_{i=1}^{d} s_{i} t_{i}$ for $s_{i}, t_{i} \in V$ if $r \neq 0$, or to be 0 if $r=0$. If we identify $V \otimes V$ with $\operatorname{Hom}\left(V^{*}, V\right)$ in a natural way, then the rank of an element in $V \otimes V$ is equal to the rank of the corresponding linear map in $\operatorname{Hom}\left(V^{*}, V\right)$. For a non-zero subspace $R \subset V \otimes V$, define the rank of $R$ to be the minimal rank of non-zero elements in $R$. Let $T(V) /(R)$ denote the quadratic algebra with relation vector space $R$. $V \otimes V$

THEOREM 0.1. Let $A$ be the quadratic algebra $T(V) /(R)$ for some $R$ with $0 \neq R \subset$

(1) If rank $R \geq \operatorname{dim} R+1$, then

(a) $A$ is Koszul of global dimension 2 ,

(b) the Hilbert series of $A$ is $H_{A}(t)=\left(1-\operatorname{dim} V t+\operatorname{dim} R t^{2}\right)^{-1}$.

Furthermore, if $R \cap W \otimes V=0$, for some subspace $W \subset V$, then

(c) $W A \cong W \otimes A$ as a right $A-$ module, and hence the subalgebra $k[W]$ is free.

(2) If $\operatorname{rank} R \geq \operatorname{dim} R+2$, then $A$ is a domain and has no non-trivial normal elements.

For basic definitions such as Koszul, global dimension and so on, see [3].

1. Hypotheses. The main idea here is induction and the hypotheses ( $\mathrm{H} 1)$ and $(\mathrm{H} 2)$ defined below are technical to make the induction work. Let $\left\{x_{i}\right\}_{1}^{n}$ be a basis of $V$. Define projections $P_{i}: V \otimes V \rightarrow V$ by $P_{i}(r)=f_{i}$ if $r=\sum_{i} x_{i} f_{i}$. Warning: $P_{i}$ is dependent on the basis $\left\{x_{i}\right\}_{1}^{n}$. For $r \in V \otimes V$, rank $r=\operatorname{dim} \sum_{i=1}^{n} k P_{i}(r)$, which is independent of the choices of $\left\{x_{i}\right\}_{1}^{n}$. If $R$ is a subspace of $V \otimes V$, then $P_{i}(R):=\sum_{r \in R} P_{i}(r)$ is a subspace of $V$ for every $i$. From now on we fix a nonzero subspace $R \subset V \otimes V$ and let $A$ be the quadratic algebra $T(V) /(R)$. For any subspace $W \subset V$, the multiplication $W \otimes A \rightarrow W A$ defines a right $A$-module homomorphism. Let $\mathbb{L}$ denote the set of all non-zero subspaces $W \subset V$ such that $W \otimes A \rightarrow W A$ is an isomorphism. If $W \in \mathbb{L}$, then $R \cap W \otimes V=0$. Note that $\mathbb{L}$ is

Glasgow Math. J. 39 (1997) 323-332. 
closed under the operation of taking non-zero subspaces; i.e., if $W \in \mathbb{L}$ and $0 \neq W^{\prime} \subset W$, then $W^{\prime} \in \mathbb{L}$. If $A$ is a domain, then every 1-dimensional subspace $W \subset V$ is in $\mathbb{L}$. Let $\Phi$ be a non-empty collection of non-zero subspaces of $V$. We say that $\Phi$ satisfies (H1) if, for every $W \in \Phi$, there is a basis $\left\{x_{i}\right\}_{1}^{n}$ of $V$ such that $\left\{x_{i}\right\}_{1}^{s}$ is a basis of $W$ for some $s<n$, and for every non-zero subspace $R^{\prime} \subset R$,

there is $i_{0}>s$ such that $P_{i_{0}}\left(R^{\prime}\right)$ is a non-zero subspace of $W^{\prime}$ for some $W^{\prime} \in \Phi$.

If, in addition to (C1),

there is $i_{1}>s$ such that $i_{1} \neq i_{0}$ and for all $(0,0) \neq(a, b) \in k^{2}, \quad\left(a P_{i_{0}}-b P_{i_{1}}\right)\left(R^{\prime}\right) \in \mathbb{L}$,

then we say $\Phi$ satisfies (H2).

If $\Phi$ satisfies (H2), then for every $0 \neq r \in R$, the dimension of $\sum_{i>s} k P_{i}(r)$ is at least 2 . In particular, rank $r \geq 2$.

Let $\bar{\Phi}=\left\{W^{\prime} \mid 0 \neq W^{\prime} \subset W\right.$, for some $\left.W \in \Phi\right\}$. Then $\bar{\Phi}$ is closed under the operation of taking non-zero subspaces. It is routine to check that $\Phi$ satisfies (H1) (or (H2)) if and only if $\bar{\Phi}$ satisfies (H1) (or (H2)). Therefore, we may assume that $\Phi$ is closed under the operation of taking non-zero subspaces if necessary. If this is the case, then (C1) can be replaced by the following condition:

$$
\text { there is } i_{0}>s \text { such that } P_{i_{0}}\left(R^{\prime}\right) \in \Phi \text {. }
$$

Suppose that $\Phi_{i}$ satisfies (H1) (or (H2)), for all $i \in I$, where $I$ is an index set. Then the union $\bigcup_{i \in I} \Phi_{i}$ satisfies (H1) (or (H2)). Therefore, for a fixed $R$, there is a maximal $\Phi_{H 1}$ satisfying (H1) (a maximal $\Phi_{H 2}$ satisfying (H2) respectively) that contains every set $\Phi$ satisfying (H1) ((H2) respectively). It is obvious that $\Phi_{H 2} \subset \Phi_{H 1}$. In general it is difficult to determine $\Phi_{H 1}$ and $\Phi_{H 2}$, but it is relatively easy to find one $\Phi$, (if it exists), satisfying (H1) or $(\mathrm{H} 2)$.

Example 1.1. Suppose that $R$ has dimension $p$ less than $n$ and suppose that there is a basis $\left\{r_{i}\right\}_{i}^{p}$ of $R$ and a basis $\left\{x_{i}\right\}_{1}^{n}$ of $V$ such that

$$
r_{i}=\sum_{j<n} x_{j} f_{j i}+x_{n} x_{i}, \quad \forall i \leq p
$$

Let $W=\sum_{i=1}^{p} k x_{i}$. Then $\Phi:=\{W\}$ satisfies $(\mathrm{H} 1)$ for $i_{0}=n$.

We will use $=_{*}$ for equality in the (free) tensor algebra $T(V)$, use ordinary $=$ for equality in $A=T(V) /(R)$, and use the same letter $f$ for an element in $T(V)$ and for the image in $A$. Since we are working on graded algebras, we will choose elements to be homogeneous. For a graded module $M=\bigoplus_{i} M_{i}, M_{>n}$ (or $M_{<n}$ ) is defined to be $\underset{i>n}{\oplus} M_{i}$ (or $\oplus_{i<n} M_{i}$ ). The following obvious fact will be used several times.

Lemma 1.2. Let $A$ be the quadratic algebra $T(V) /(R)$, for some $0 \neq R \subset V \otimes V$. Suppose that the multiplication induces an isomorphism $W \otimes I \cong W I$, for some subspaces 
$W, I \subset A$. If $\left\{g_{j}\right\}$ is a linearly independent set of $I$ and $\left\{f_{j}\right\}$ is a subset of $W$ such that $\sum_{j} f_{j} g_{j}=0$, then $f_{j}=0$ for all $j$.

Proposition 1.3. Let $A$ be the quadratic algebra $T(V) /(R)$ for some $R$ with $0 \neq R \subset V \otimes V$ and suppose that there is a $\Phi$ satisfying (H1). Then $\Phi \subset \mathbb{L}$.

Proof. Given a $W \in \Phi$, we pick a basis $\left\{x_{i}\right\}_{1}^{n}$ as in the definition of (H1). In particular, $\left\{x_{i}\right\}_{1}^{s}$ is a basis of $W$. It suffices to show that if $\sum_{i=1}^{s} x_{i} t_{i}=0$ then $t_{i}=0$, for all $i \leq s$. Since $A$ is graded, we may assume that $t_{i}$ is either 0 or homogeneous of degree $d$, for all $i$.

If $d=0$, then $t_{i} \in k$ and hence $t_{i}=0$, because $\left\{x_{i}\right\}_{1}^{s}$ are linearly independent. If $d=1$, then $\sum_{i=1}^{s} x_{i} t_{i}=0$ implies that $\sum_{i=1}^{s} x_{i} t_{i}={ }_{*} r^{\prime} \in R$. For any $i_{0}>s, P_{i_{0}}\left(r^{\prime}\right)={ }_{*} P_{i_{0}}\left(\sum_{i=1}^{s} x_{i} t_{i}\right)={ }_{*} 0$. By (C1), $r^{\prime}=0$. Hence $t_{i}={ }_{*} P_{i}\left(\sum_{i=1}^{s} x_{i} t_{i}\right)={ }_{*} P_{i}\left(r^{\prime}\right)={ }_{*} 0$ for all $i \leq s$.

Now suppose that $d>1$. If $\sum_{i=1}^{s} x_{i} t_{i}=0$, then

$$
\sum_{i=1}^{s} x_{i} t_{i}=* \sum f_{w}^{u} r^{u} g_{w}^{u}+\sum_{j=1}^{q} r_{j} g_{j},
$$

for some $r^{u}, r_{j} \in R, f_{w}^{u}, g_{j} \in T(V)_{>0}$ and $g_{w}^{u} \in T(V)$. We assume that $q$ in (E1) is as small as possible. Write $f_{w}^{u}={ }_{*} \sum_{i} x_{i} f_{i w}^{u}$ and $r_{j}=* \sum_{i} x_{i} r_{i j}$. Hence

$$
\begin{array}{ll}
t_{i}=* \sum f_{i w}^{u} r^{u} g_{w}^{u}+\sum_{j=1}^{q} r_{i j} g_{j}, & \forall i \leq s, \\
0=* \sum f_{i w}^{u} r^{u} g_{w}^{u}+\sum_{j=1}^{q} r_{i j} g_{j}, & \forall i>s .
\end{array}
$$

Passing to $A$ we have $t_{i}=\sum_{j=1}^{q} r_{i j} g_{j}$ for $i \leq s$ and $0=\sum_{j=1}^{q} r_{i j} g_{j}$ for $i>s$. If $q>0$, then $\left\{g_{j}\right\}$ are linearly independent in $A$. By (C1), there is $i_{0}>s$ such that $P_{i_{0}}\left(\sum_{i=1}^{q} k r_{j}\right)$ is a non-zero subspace of $W^{\prime}$, for some $W^{\prime} \in \Phi$. By induction, $W^{\prime} A_{<d} \cong W^{\prime} \otimes A_{<d}$. By Lemma 1.2, $0=\sum_{j=1}^{q} r_{i_{0} j} g_{j}$ implies that $r_{i_{0} j}=0$ for all $j$. This contradicts the fact that $P_{i_{0}}\left(\sum_{j=1}^{q} k r_{j}\right)$ is non-zero. Therefore $q=0$. It follows from (E2) that $t_{i}=0$ for all $i \leq s$, as desired.

The following is an immediate consequence of Lemma 1.2 and Proposition 1.3.

Corollary 1.4. Let $A$ be the quadratic algebra $T(V) /(R)$, for some $R$ with $0 \neq R \subset V \otimes V$. Suppose that there exists a $\Phi$ satisfying (H1). Let $W=\sum_{i=1}^{s} k x_{i}$ be in $\Phi$, where $\left\{x_{i}\right\}_{i=1}^{n}$ is a basis of $V$. Let $\left\{g_{j}\right\}_{q} \subset A$ and $\left\{r_{j}\right\}_{\{} \subset R$ be linearly independent sets of 
elements, and write $r_{j}=* \sum_{i=1}^{n} x_{i} r_{i j}$. Then, if $i_{0}>\dot{s}$ is as in $(\mathrm{C} 1)$, one has $\sum_{j=1}^{q} r_{i_{0} j} g_{j} \neq 0$. In particular, if $a_{i_{0}} g=\sum_{j=1}^{q} e_{i_{0} j} g_{j}$, for some $a_{i_{0}}$ and $g$, then $a_{i_{0}} \neq 0$.

Proposition 1.3 shows that $\Phi_{H 1} \subset \mathbb{L}$. We shall see that $\Phi_{H 1}=\mathbb{L}$ if $\operatorname{rank} R \geq \operatorname{dim} R+1$.

ExAmple 1.5. (1) Let $p \leq n / 2-1$ and let $\left\{x_{i}\right\}_{1}^{n}$ be a basis of $V$. Suppose that $R \subset V \otimes V$ has a basis

$$
r_{i}=\sum_{j \leq n-2} x_{j} f_{j i}+x_{n-1} x_{i}+x_{n} x_{p+i}, \quad \forall i \leq p
$$

Let $W=\sum_{i \leq 2 p} k x_{i}$. Then $\Phi:=\{W\}$ satisfies (H1) for $i_{0}=n$. By Proposition 1.3, $W \in \mathbb{L}$. Using this fact it is easy to check that $\Phi$ satisfies (H2) for $i_{0}=n$ and $i_{1}=n-1$.

(2) Suppose that $V=W \oplus W^{\prime}$ with $W, W^{\prime} \neq 0$ and $0 \neq R \subset W^{\prime} \otimes W$. Pick a basis $\left\{x_{i}\right\}_{1}^{n}$ of $V$ such that $\left\{x_{i}\right\}_{1}^{s}$ is a basis of $W$ and $\left\{x_{i}\right\}_{s+1}^{n}$ is a basis of $W^{\prime}$. Set $\Phi=\{W\}$; then $\Phi$ clearly satisfies (H1). Similarly, for all $i_{0}, i_{1}>s$ and all $a, b \in k,\left(a P_{i_{0}}-b P_{i_{1}}\right)\left(R^{\prime}\right) \subset W$. By Proposition 1.3, $W$ is in $\mathbb{L}$ and hence every non-zero subspace of $W$ is in $\mathbb{L}$.

If, moreover, rank $R \geq 2$, then, for every $0 \neq R^{\prime} \subset R$, there are $i_{0}, i_{1}>s$ and $i_{0} \neq i_{1}$ such that, for all $(0,0) \neq(a, b),\left(a P_{i_{0}}-b P_{i_{1}}\right)\left(R^{\prime}\right) \neq 0$. Hence $\left(a P_{i_{0}}-b P_{i_{1}}\right)\left(R^{\prime}\right) \in \mathbb{L}$ and thus $\Phi$ satisfies $(\mathrm{H} 2)$.

2. Proof of Theorem 0.1. For an integer $l$ the shift module $M(l)$ of a graded module $M=\bigoplus_{i \in \mathbf{Z}} M_{i}$ is defined by $M(l)_{i}=M_{i+l}$. If $\operatorname{dim} M_{i}<\infty$ for all $i$, the Hilbert Series of $M$ is defined to be $H_{M}(t)=\sum_{i} \operatorname{dim} M_{i} t$. The trivial $A$-module $A / A_{>0}$ is denoted by $k_{A}$.

THEOREM 2.1. Let $A$ be a quadratic algebra $T(V) /(R)$ and suppose that there is a basis $\left\{x_{i}\right\}_{1}^{n}$ such that, for every non-zero subspace $R^{\prime} \subset R, P_{i}\left(R^{\prime}\right) \in \mathbb{L}$, for some $i$. Then

(1) $k_{A}$ has a minimal projective resolution

$$
0 \rightarrow A(-2)^{\operatorname{dim} R} \rightarrow A(-)^{\operatorname{dim} v} \rightarrow A \rightarrow k_{A} \rightarrow 0,
$$

(2) $A$ is a Koszul algebra of global dimension 2,

(3) $H_{A}(t)=\left(1-\operatorname{dim} V t+\operatorname{dim} R t^{2}\right)^{-1}$.

As a consequence, if there is a $\Phi$ satisfying (H1), then (1), (2), (3) hold.

Proof. Consider the exact sequence

$$
A(-2)^{\operatorname{dim} R} \rightarrow A(-1)^{\operatorname{dim} v} \rightarrow A \rightarrow k_{A} \rightarrow 0
$$

constructed by using generators and relations. Pick a basis $\left\{r_{j}\right\}_{j=1}^{p}$ of $R$. Then the boundary map $\partial_{2}: A(-2)^{\operatorname{dim} R} \rightarrow A(-1)^{\operatorname{dim} V}$ can be represented as a left multiplication by a matrix $B:=\left(r_{i j}\right)$, where $r_{j}=* \sum_{i} x_{i} r_{i j}$. We claim that $\partial_{2}$ is injective; namely, if $B\left(g_{1}, \ldots, g_{p}\right)^{\tau}=0$, then $g:=\left(g_{1}, \ldots, g_{p}\right)=0$. If $g \neq 0$, pick a basis for $\left\{g_{j}\right\}_{1}$, say $\left\{g_{j}^{\prime}\right\}_{1}^{\nu}$, and write $g^{\tau}=N g^{\prime \tau}$ for a non-zero $p \times v$ matrix $N$ over $k$. Then $B N g^{\prime \tau}=0$. Let $R^{\prime}$ be the subspace generated by the components of $\left(r_{1}^{\prime}, \ldots, r_{v}^{\prime}\right):={ }_{*}\left(r_{1}, \ldots, r_{p}\right) N==_{*}\left(x_{1}, \ldots, x_{n}\right) B N$. Then $R^{\prime}$ is non-zero. 
Write $r_{j}^{\prime}=\sum_{j=1}^{n} x_{i} r_{i j}^{\prime}$; then $\sum_{j=1}^{v} r_{i j}^{\prime} g_{j}^{\prime}=0$ for all $i$. By the hypothesis there is $i$ such that $P_{i}\left(R^{\prime}\right) \in \mathbb{L}$. By Lemma $1.2, r_{i j}^{\prime}=0$ for all $j$. This contradicts the fact that $P_{i}\left(R^{\prime}\right)$ is non-zero. Therefore $\partial_{2}$ is injective and hence (E4) is a minimal projective resolution of $k_{A}$.

The projective resolution (E4) is linear in the sense of [3, Definition 1.6], whence the algebra $A$ is Koszul [3, Definition 5.4]. The global dimension of $A$ is equal to the projective dimension of $k_{A}$, equal to 2 . Since dim is additive on exact sequences, it follows from (E4) that

$$
H_{A}(t)\left(1-\operatorname{dim} V t+\operatorname{dim} R t^{2}\right)=H_{k_{A}}(t)=1 .
$$

Thus $H_{A}(t)=\left(1-\operatorname{dim} V t+\operatorname{dim} R t^{2}\right)^{-1}$.

If there is a $\Phi$ satisfying (H1) then, by Proposition 1.3, the hypothesis holds for $i=i_{0}$ appearing in (C1). Therefore (1), (2), (3) hold.

A simple case is when $\operatorname{dim} V=3$ and

$$
\begin{aligned}
& r_{1}=a x_{1}^{2}+b x_{2} x_{3}+c x_{3} x_{2}, \\
& r_{2}=c x_{1} x_{3}+a x_{2}^{2}+b x_{3} x_{1}=a x_{2}^{2}+b x_{3} x_{1}+c x_{1} x_{3},
\end{aligned}
$$

where $a, b, c$ are non-zero scalars in $k$. By Example 1.1, $\Phi=\left\{k x_{1}+k x_{2}\right\}$ satisfies (H1) and, by Theorem 2.1, $T(v) /\left(r_{1}, r_{2}\right)$ is a Koszul algebra of global dimension 2 . Note that $T(V) /\left(r_{1}, r_{2}\right)$ is isomorphic to $k\left\langle x_{1}, x_{2}\right\rangle\left[x_{3}, \sigma, \delta\right]$, for some automorphism $\sigma$ and some $\sigma$-derivation $\delta$ of the free algebra $k\left\langle x_{1}, x_{2}\right\rangle$, whence it is a domain. By adding another relation $r_{3}=a x_{3}^{2}+b x_{1} x_{2}+c x_{2} x_{1}=0$, we obtain the type- $A$ algebra of global dimension 3 studied in [2]. For the algebra $T(V) /\left(r_{1}, r_{2}, r_{3}\right)$, there is no $\Phi$ satisfying (H1) because its global dimension is not 2 .

Proof of Theorem 0.1(1). Suppose that $\operatorname{rank} R \geq \operatorname{dim} R+1=p+1$. Let $\Phi$ be the set of all non-zero subspaces of $V$ of dimension at most $p$. Given a $W \in \Phi$, we pick a basis $\left\{x_{i}\right\}_{1}^{n}$ of $V$ such that $\left\{x_{i}\right\}_{1}^{s}$ is a basis of $W$. For every $0 \neq R^{\prime} \subset R, P_{i}\left(R^{\prime}\right)$ has dimension $p$ or less. Since rank $R^{\prime} \geq \operatorname{rank} R>p$, there is $i_{0}>p \geq s, P_{t_{0}}\left(R^{\prime}\right) \neq 0$; and hence $P_{t_{0}}\left(R^{\prime}\right) \in \Phi$. Thus $\Phi$ satisfies (H1), and (a) and (b) of Theorem 0.1(1) follows from Theorem 2.1.

To prove (c) we let $W$ be a subspace of $V$ such that $R \cap W \otimes V=0$. Pick a basis $\left\{x_{i}\right\}_{1}^{n}$ such that $\left\{x_{i}\right\}_{1}^{5}$ is a basis of $W$. Assume that $\sum_{i \leq s} x_{i} t_{i}=0$. The notation being as in (E1-3), we obtain, in a similar way, that

and that

$$
\sum_{i \leq s} x_{i} t_{i}=* \sum f_{w}^{u} r^{u} g_{w}^{u}+\sum_{j=1}^{q} r_{j} g_{j}
$$

$$
\begin{array}{ll}
t_{i}={ }_{*} \sum f_{i w}^{u} r^{u} g_{w}^{u}+\sum_{j=1}^{q} r_{i j} g_{j}, & \forall i \leq s, \\
0=* \sum f_{i w}^{u} r^{\mu} g_{w}^{u}+\sum_{j=1}^{q} r_{i j} g_{j}, & \forall i>s .
\end{array}
$$

We pick $q$ as small as possible. If $q>0$, then $q \leq p$ and $\left\{g_{1}, \ldots, g_{q}\right\}$ are linearly independent in $A$. Since $R \cap W \otimes V=0$, there is $i_{0}>s$ such that $W^{\prime}:=P_{i_{0}}\left(\sum_{j=1}^{q} k r_{j}\right) \neq 0$. Hence $W^{\prime}$ is in $\Phi$ and, by Proposition 1.3, $W^{\prime}$ is in L. Passing to $A$, (E5) implies that 
$0=\sum_{j} r_{i_{j} j} g_{j}$. This contradicts Lemma 1.2. Hence $q=0$ and so $t_{i}=0$, for all $i \leq s$. Therefore $W \in \mathbb{L}$, as desired.

By the proof of (c) above we also see that if $\operatorname{rank} R \geq \operatorname{dim} R+1$ then

$$
\Phi_{H 1}=\mathbb{L}=\{W \mid 0 \neq W \subset V \text { and } R \cap W \otimes V=0\} .
$$

THEOREM 2.2. Let $A$ be a quadratic algebra $T(V) /(R)$ with a $\Phi$ satisfying $(\mathrm{H} 2)$.

(1) $A$ is a domain.

(2) Let $f, g \in A_{>0}-\{0\}$. If $f g \in W A$, for some $W \in \Phi$, then $f \in W A$.

Proof. By Proposition 1.3, $W A \cong W \otimes A$, for all $W \in \Phi$. Without loss of generality, we may assume that $\Phi$ is closed under the operation of taking non-zero subspaces, whence we can use $\left(\mathrm{Cl}^{\prime}\right)$ instead of $(\mathrm{C} 1)$. Since $A$ is $\mathbb{N}$-graded, it suffices to prove the following statement:

(\#) Let $f$ and $g$ be homogeneous elements in $A_{>0}-\{0\}$ and let $W \in \Phi$. Then (\#a) $f g \neq 0$, and $(\# b)$ if $f g \in W A$, then $f \in W A$.

We prove (\#) by induction on $m:=\operatorname{deg} f+\operatorname{deg} g$.

Initial step: $m=2$.

(\#a) Assume on the contrary that $f g=0$. Then $f g={ }_{*} r^{\prime} \in R$. Since $f, g \neq * 0$ in $T(V)$, $r^{\prime} \neq 0$ and rank $r^{\prime}=\operatorname{rank} f g=1$. This contradicts (H2). Therefore $f g \neq 0$ in $A$.

(\#b) If $f g \in W A$, then $f g={ }_{*} r^{\prime}+\sum_{i \leq s} x_{i} h_{i}$ in the free algebra $T(V)$, where $\left\{x_{i}\right\}_{1}^{s}$ is a basis of $W$. Suppose that $r^{\prime} \neq 0$. Write $f={ }_{*} \sum_{i} x_{i} a_{i}$ and $r^{\prime}={ }_{*} \sum_{i} x_{i} r_{i}^{\prime}$; then we have $a_{i} g={ }_{*} r_{i}^{\prime}+h_{i}$, for all $i \leq s$, and $a_{i} g={ }_{*} r_{i}^{\prime}$, for all $i>s$. Thus $\operatorname{dim} \sum_{i>s}^{i} k P_{i}\left(r^{\prime}\right) \leq \operatorname{dim} k g \leq 1$. This contradicts (H2). Therefore $r^{\prime}=0$ and $f \in \sum_{i \leq s} x_{i} T(V)$, whence $f \in W A$.

Inductive step: $m>2$.

$(\# b)$ For any $W \in \Phi$, pick a basis $\left\{x_{i}\right\}_{1}^{n}$ of $V$ as in (H2). In particular, $\left\{x_{i}\right\}_{1}^{S}$ is a basis of $W$. If $f g \in W A$ then, similarly to (E1), $f g=* \sum f_{w}^{u} r^{u} g_{w}^{u}+\sum_{j=1}^{q} r_{j} g_{j}+\sum_{i \leq s} x_{i} h_{i}$, for some $f_{w}^{u}, g_{j}$, $h_{i} \in T(V)_{>0}$. We choose a preimage $f$ in $T(V)$ such that $q$ is as small as possible. Write $f=* \sum_{i} x_{i} a_{i}, f_{w}^{u}=* \sum_{i} x_{i} f_{i w}^{u}$ and $r_{j}=* \sum_{i} x_{i} r_{i j}$. Then

$$
\begin{aligned}
& a_{i} g=* \sum f_{i w}^{u} r^{u} g_{w}^{u}+\sum_{j=1}^{q} r_{i j} g_{j}+h_{i}, \quad \forall i \leq s, \\
& a_{i} g=* \sum f_{i w}^{u} r^{u} g_{w}^{u}+\sum_{j=1}^{q} r_{i j} g_{j}, \quad \forall i>s .
\end{aligned}
$$

Passing to $A$, we have $a_{i} g=\sum_{j=1}^{q} r_{i j} g_{j}$, for all $i>s$. Assume that $q>0$. Then $\left\{g_{j}\right\} q$ are 
linearly independent in $A$ and $\left\{r_{i}\right\}_{=1}^{q}$ are linearly independent in $R$. By Corollary 1.4, $a_{i_{0}} \neq 0$ for $i_{0}$ as in (C1).

If $a_{i_{0}}$ has degree 0 (or $\left.\operatorname{deg} f=1\right)$ then, for $i_{0}$ and $i_{1}$ as in (C2),

$$
0=a_{i_{0}} a_{i_{1}}(g-g)=a_{i_{1}}\left(a_{i_{0}} g\right)-a_{i_{0}}\left(a_{i} g\right)=\sum\left(a_{i_{1}} r_{i_{0} j}-a_{i_{0}} r_{i_{1}}\right) g_{j} \text {. }
$$

By (C2), $\left(a_{i_{1}} P_{i_{0}}-a_{i_{0}} P_{i_{1}}\right)\left(\sum_{j=1}^{q} k r_{j}\right) \in \mathbb{L}$. By Lemma 1.2, (E6) implies that $a_{i_{1}} r_{i_{0} j}-a_{i_{0}} r_{i_{1} j}=0$, for all $j$, which contradicts that $\left(a_{i_{1}} P_{i_{0}}-a_{i_{0}} P_{i_{1}}\right)\left(\sum_{j=1}^{q} k r_{j}\right) \neq 0$, as stated in (C2). Next we consider the case when the degree of $a_{i_{0}}$ is positive. By $\left(\mathrm{Cl}^{\prime}\right), W^{\prime}:=\sum_{j=1}^{q} k r_{i_{0} j}$ is in $\Phi$. Without loss of generality, we suppose that $\left\{r_{i_{0}}\right\}_{1}^{l}$ is a basis of $W^{\prime}$, for some $l \leq q$. Hence $a_{i_{0}} g \in W^{\prime} A \cong W^{\prime} \otimes A$. By the induction hypothesis $(\# b), a_{i_{0}}=\sum_{j=1}^{l} r_{i_{0} j} w_{j}$, for some $w_{j} \in A$. Replacing $f$ by $f-\sum_{j=1}^{l} r_{j} w_{j}$ (which is equal to $f$ in $A$ ), we obtain $a_{i_{0}}=0$ for this new $f$. (Note that replacing $f$ by $f-\sum_{j=1}^{l} r_{j} w_{j}$ will not change $\left\{r_{j}\right\}$ because $q$ is the smallest possible, but will change $\left\{g_{j}\right\}_{1}^{q}$ obviously.) This contradicts $a_{i_{0}} \neq 0$, proved in the end of the last paragraph. Therefore we must reject the assumption that $q>0$. Hence $q=0$. As a consequence $a_{i} g=0$ in $A$ and so $a_{i}=0$, for all $i>s$, by the induction hypothesis (\#a). Therefore $f=\sum_{i \leq s} x_{i} a_{i} \in W A$, as required.

(\#a) Assume on the contrary that $f g=0$. Then $f g \in W A$ for any $W=\Phi$. By $(\# b)$ proved above, $f \in W A$; i.e., $f=\sum_{i=1}^{s} x_{i} a_{i}$. Hence $0=f g=\sum_{i=1}^{s} x_{i} a_{i} g$. Since $W A \cong W \otimes A$, $a_{i} g=0$, for all $i \leq s$. By the induction hypothesis $(\# a), a_{i}=0$ for all $i \leq s$, whence $f=\sum_{i=1}^{s} x_{i} a_{i}=0$. This contradicts $f \neq 0$. Therefore $f g \neq 0$.

EXAMPLE 2.3. Let $r=x_{1} y_{1}+x_{2} y_{2} \in V \otimes V$ be an element of rank 2 and $R$ the 1-dimensional subspace $k r$. Hence $\operatorname{rank} R=\operatorname{dim} R+1$. By Theorem $0.1(1 \mathrm{c})$, every 1-dimensional subspace of $V$ is in $\mathbb{Q}$. Suppose that $k y_{1}+k y_{2} \neq k x_{1}+k x_{2}:=W_{x}$. Then either $y_{1} \notin W_{x}$ or $y_{2} \notin W_{x}$. Let $\Phi$ be the set of all 1-dimensional subspaces of $V$ not contained in $W_{x}$. It is easy to check that $\Phi$ satisfies (H2). By Theorem $2.2, T(V) /(r)$ is a domain.

Proposition 2.4. Let $A$ be a quadratic algebra $T(V) /(R)$ and $\operatorname{dim} V>1$. Suppose that there is a right regular element $x \in V$ such that if $f g \in x A$, for some $f, g \in A_{>0}-\{0\}$, then $f \in x A$. Then $A$ has no non-trivial normal elements.

Proof. Assume the contrary and let $g$ be a non-trivial normal element. Since $A$ is graded we may assume that $g$ is homogeneous of position degree. Since $g$ is normal, there is $y \in V$ such that $g y=x g \in x A$. By the hypothesis, $g=x g_{0}$ and hence $x g_{0} y=x x g_{0}$. Therefore $g_{0} y=x g_{0}$ by cancelling the right regular element $x$. It follows by induction that $y=x$ and $g=l x^{n}$, for some nonzero scalar $l$ and some positive integer $n$. Hence $x^{n}$ is normal. Pick an element $h \in V-k x$. By the hypothesis, $h x^{n} \notin x A$, which contradicts the fact that $x^{n}$ is normal. Therefore $A$ has no non-trivial normal element. 
Proof of Theorem 0.1(2). Suppose that $\operatorname{rank} R \geq \operatorname{dim} R+2=p+2$. Let $\Phi$ be the set of all non-zero subspaces of $V$ of dimension at most $p$. We shall prove that $\Phi$ satisfies (H2). By the proof of Theorem 0.1(1), $\Phi$ satisfies (H1) and Proposition 1.3, $\Phi \subset \mathbb{L}$. It remains to check $(\mathrm{C} 2)$.

For every $W \in \Phi$, pick a basis $\left\{x_{i}\right\}_{i=1}^{n}$ of $V$ such that $\left\{x_{i}\right\}_{i=1}^{s}$ is a basis of $W$. For every $i_{0}, i_{1}>s, i_{0} \neq i_{1}$ and every $(a, b) \in k^{2},\left(a P_{i_{0}}-b P_{i_{1}}\right)(R)$ is a subspace of dimension at most $p$. It suffices to show that for every $0 \neq r \in R$, there exist $i_{0}, i_{1}>s$ such that for every $(0,0) \neq(a, b) \in k^{2}, \quad\left(a P_{i_{0}}-b P_{i_{1}}\right)(r) \neq 0$. This follows immediately from the fact that $\operatorname{rank} r \geq \operatorname{rank} R \geq p+2$. Hence $\Phi$ satisfies (H2). By Theorem 2.2(1), $A$ is a domain. By Theorem 2.2(2), the hypothesis of Proposition 2.4 holds for every nonzero element $x$ in $V$. Therefore $A$ has no non-trivial normal element.

$$
\begin{aligned}
& \text { If rank } R \geq \operatorname{dim} R+2 \text {, then } \\
& \qquad \Phi_{H 2}=\{W \mid 0 \neq W \subset V \text { and } R \cap(W+k x) \otimes V=0 \text { for all } x \in V\} .
\end{aligned}
$$

\section{Some remarks.}

REMARK 3.1. The rank of a single element of $V \otimes V$ is preserved under field extension, but the rank of a subspace of $V \otimes V$ is not.

Let $V$ be a 4-dimensional space over the real numbers $\mathbb{R}$ with a basis $\left\{x_{1}, x_{2}, x_{3}, x_{4}\right\}$ and let $r_{1}=x_{1} x_{4}+x_{2} x_{3}$ and $r_{2}=x_{1} x_{3}-x_{2} x_{4}$. Then $R:=\mathbb{R} r_{1}+\mathbb{R} r_{2}$ has rank 2 and $R \subset W^{\prime} \otimes W$ with $W=\mathbb{R} x_{1}+\mathbb{R} x_{2}$ and $W^{\prime}=\mathbb{R} x_{3}+\mathbb{R} x_{4}$. By Example 1.5(2), $\Phi=\{W\}$ satisfies (H2) and, by Theorem $2.2(1), T(V) /(R)$ is a domain. After extending $\mathbb{R}$ to complex numbers $\mathbb{C}$, the rank of the relations $R \otimes \mathbb{C}$ becomes 1 because

$$
\left(x_{1}+i x_{2}\right)\left(x_{3}+i x_{4}\right)=x_{1} x_{3}-x_{2} x_{4}+i\left(x_{1} x_{4}+x_{2} x_{3}\right) \in R \otimes \mathbb{C} \text {. }
$$

Hence $A \otimes \mathbb{C}$ is not a domain. In particular, there are no $\Phi$ satisfying (H2). Therefore extending the base field does not preserve the hypothesis $(\mathrm{H} 2)$ generally.

Remark 3.2. Suppose that $\Phi$ satisfies (H1) for $T(V) /(R)$. Let $R^{\prime}$ be a non-zero subspace of $R$. Then it is easy to see that $\Phi$ satisfies (H1) for $T(V) /\left(R^{\prime}\right)$. As a consequence, $T(V) /\left(R^{\prime}\right)$ is Koszul of global dimension 2, for all $R^{\prime}$ with $0 \neq R^{\prime} \subset R$.

REMARK 3.3. If $k$ is an infinite field and $R$ is a generic subspace of $V \otimes V$ of dimension less than $n:=\operatorname{dim} V$, then $T(V) /(R)$ is Koszul of global dimension 2, as we will now show. The Koszul property and global dimension are preserved by extending the field, because the minimal projective resolution of the trivial module $k_{A}$ remains easily the same. Hence, since $k$ is infinite, we can replace it by its algebraic closure. By Theorem 2.1, it suffices to show that there exists a $\Phi$ satisfying (H1). By Remark 3.2, we only need consider the case when $R$ has dimension $n-1$. Pick $n-1$ generic elements in $V \otimes V$ as basis elements of $R$

$$
r_{s}=\sum_{i j} a_{s i j} x_{i} x_{j} \quad(s=1, \ldots, n-1) .
$$

Since $k$ is algebraically closed, after changing a basis for $V$ we may assume that $a_{s n n}=0$, for all $s$. Hence $R$ has a basis consisting of elements

$$
r_{s}=\sum_{i<n} a_{s i j} x_{i} x_{j}+x_{n} x_{s} \quad(s=1, \ldots, n-1) .
$$

By Example 1.1, there is a $\Phi$ satisfying (H1) as desired. 
REMARK 3.4. Let $k$ be an infinite field and $R$ a $d$-dimensional subspace of $V \otimes V$ with $1 \leq d<n$. Then $T(V) /(R)$ is generically a domain. We sketch a proof next and details are left to the interested reader. If $A \otimes K$ is a domain with field extension $K$ over the base field $k$, then $A$ itself is a domain. By the argument of Remark 3.3, we may assume $k$ is algebraically closed. After changing the basis $\left\{x_{i}\right\}_{1}^{n}$ of $V$ we may assume that $R$ has a basis $\left\{r_{s}\right\}_{1}^{d}$ and that

$$
r_{s}=\sum_{i<n} a_{s i j} x_{i} x_{j}+x_{n} x_{s} \quad(s=1, \ldots, d) .
$$

By Remark 3.3 and Theorem 2.1, $A=T(V) /(R)$ is Koszul of global dimension 2 and the Hilbert series of $A$ is $\left(1-\operatorname{dim} V t+\operatorname{dim} R t^{2}\right)^{-1}$. In particular, $A$ has a $k$-linear monomial basis

$$
\left\{x_{i_{1}} \ldots x_{i_{l}} \mid \text { for all }\left(i_{1}, \ldots, i_{l}\right) \text {, and for } 1 \leq p<l,\left(i_{p}, i_{p+1}\right) \neq(n, s) \text { for any } s \leq d\right\} \text {. }
$$

Consider the $\mathbb{N}$-filtration of $A$ induced by $\operatorname{deg} x_{i}=1$, for all $n>i>d$, $\operatorname{deg} x_{i}=2$, for all $i \leq d$ and $\operatorname{deg} x_{n}=3$, and let $\operatorname{gr}(A)$ be the associated graded ring. By definition $\operatorname{gr}(A)$ is a factor ring of $C:=k\left\langle x_{i}\right\rangle /\left(\bar{r}_{1}, \ldots, \bar{r}_{d}\right)$, where $\bar{r}_{s}=\sum_{i=d} a_{\sin } x_{i} x_{n}+x_{n} x_{s}$. It is easy to see that $C$ is an extension of $k\left\langle x_{1}, \ldots, \ldots x_{d}, x_{n}\right\rangle /\left(\bar{r}_{s}\right)$ by adding $x_{d+1}, \ldots, x_{n-1}$ freely. Hence $C$ has a monomial basis (E7). Therefore $\operatorname{gr}(A)$ is isomorphic to $C$. Since $k\left(x_{1}, \ldots, \ldots x_{d}, x_{n}\right) /\left(\bar{r}_{s}\right)$ is isomorphic to $k\left\langle x_{1}, \ldots, x_{d}\right\rangle\left[x_{n}, \sigma\right]$, for some automorphism $\sigma$ (generically), it is a domain. Therefore $C$ is a domain and hence $A$ is a domain.

Remark 3.5. Let $k$ be an infinite field and $1 \leq d<n=\operatorname{dim} V$. By linear algebra, a generic $d$-dimensional subspace $R \subset V \otimes V$ has rank at least $n-\lfloor\sqrt{d-1}\rfloor$ If $k$ is algebraically closed, then the rank of a generic $d$-dimensional subspace $R$ is $n-\lfloor\sqrt{d-1}\rfloor$ (proved by S. P. Smith). As a consequence, if $k$ is an infinite field and $d \leq\left(\sqrt{n-\frac{3}{4}}-\frac{1}{2}\right)^{2}$, then a generic $d$-dimensional vector space $R$ has rank at least $d+2$. By Theorem 0.1(2), $A=T(V) /(R)$ is a domain. (This is another way to prove the result in Remark 3.4 when $d \leq\left(\sqrt{n-\frac{3}{4}}-\frac{1}{2}\right)^{2}$.)

Remark 3.6. S. P. Smith and the author [4] and [5] proved the followng result for one-relator quadratic algebras, some of which was based on this note. Parts (1) and (2) are well known. Then

Let $A$ be a one-relator quadratic algebra $T(V) /(r)$ for some $r$ with $0 \neq r \in V \otimes V$.

(1) $A$ is Koszul;

(2) $\operatorname{gl} \cdot \operatorname{dim} A= \begin{cases}\infty & \text { if } r=x^{2}, \text { for some } x \in V \text {, } \\ 2 & \text { otherwise; }\end{cases}$

(3) $A$ is noetherian if and only if rank $r=\operatorname{dim} V=2$;

(4) $A$ is a domain if and only if rank $r \geq 2$;

(5) if $A$ has a non-trivial normal element, then $\operatorname{dim} V=\operatorname{rank} r=2$;

(6) if $W \subset V$ and $r \notin W \otimes V$, then the multiplication $W \otimes A \rightarrow W A$ induces an $A$-module isomorphism, and hence the subalgebra $k[W]$ is free.

Acknowledgement. The author would like to thank M. Artin, J. T. Stafford and S. P. Smith for several useful comments. 


\section{REFERENCES}

1. D. J. Anick, On the homology of associated algebras, Trans. Amer. Math. Soc. 296 (1986) No. 2, 641-659.

2. M. Artin, J. Tate and M. van den Bergh, Some algebras associated to automorphisms of elliptic curves, in The Grothendieck Festschrift (P. Cartier et al. Editors) Vol. 1 (Birkhauser, 1990), 33-85.

3. S. P. Smith, Some finite dimensional algebras related to elliptic curves, in Representation theory of algebras and related topics, Volume 19, CMS Conference Proceedings, (Canadian Math. Soc., 1996), 315-348.

4. S. P. Smith and J. J. Zhang, Regular algebras and Koszul algebras, preprint, 1996. appear.

5. J. J. Zhang, Non-Noetherian regular rings of dimension 2, Proc. Amer. Math. Soc., to

\section{Department of Mathematics}

BOX 354350

UNIVERSITY OF WASHINGTON

SEATTLE

WASHINGTON 98195

U.S.A.

E-mail: zhang@math.washington.edu 